\title{
Intervenciones para la cesación tabáquica
}

\author{
Smoking cessation interventions
}

Ranney L y col. Ann Intern Med 2006; 145: 84556

\section{Objetivo}

Revisar estrategias y evaluar su eficacia en el tratamiento de cesación tabáquica en adultos y poblaciones especiales.

\section{Fuente de datos}

MEDLINE, Cumulative Index to Nursing and Applied Health (CINAHL) Cochrane Library, Cochrane Clinical Trials Register, Psychological Abstracts, and Sociological Abstracts (1980 a 2005).

\section{Seleccion de estudios}

Revisiones sistemáticas, estudios aleatorizados, controlados y observacionales

\section{Extracción de datos}

Dos investigadores analizaron los trabajos por separado respecto de su diseño, población incluida, tamaño de muestra, tratamiento estadístico, resultados y calidad

\section{Resultados principales}

Se hallaron 1288 artículos, se examinaron 488 potenciales a incluir y solo se incluyeron 102 (siete examinaron intervenciones en pacientes con condiciones especiales como problemas psiquiátricos y abuso de drogas).

Las estrategias de autoayuda son ineficaces cuando son usadas en forma aislada. El consejo o la intervención breve y los tratamientos farmacológicos pueden mejorar la tasa de éxito (14 estudios proporcionaron pruebas suficientes de la eficacia de la farmacoterapia).
Pocos estudios se centraron en el modo de ayudar o tratar a poblaciones especiales. Los tres estudios con pacientes hospitalizados obtuvieron conclusiones coincidentes: el diagnóstico clínico no modificaba la probabilidad de éxito. La evidencia fue insuficiente en cuanto a la eficacia de las intervenciones para pacientes psiquiátricos y en pacientes con problemas de abuso de drogas.

\section{Conclusiones \\ La presente revisión mostró resultados similares a las anterio- res. Se recomiendan las intervenciones breves asociadas o no a los fármacos disponibles como sustitutos de nicotina, bupro- pion $u$ otros antidepresivos (no hay suficiente evidencia sobre la efectividad de otros antidepresivos) y la combinación de estos fármacos, según se requiera, en pacientes que decidan dejar de fumar. \\ La utilización de tratamientos eficaces para dejar de fumar está totalmente indicada en todas las poblaciones y especialmente en los grandes fumadores, en las madres durante el post parto inmediato, en los pacientes psiquiátricos y en las poblaciones con historia de abuso de drogas. Los nuevos estudios no resolvieron las inconsistencias existentes acerca de la eficacia de aumentar la intensidad del asesoramiento y/o los tratamien- tos farmacológicos combinados en los pacientes con antecedente de fracaso en tratamientos menos intensos.}

Palabras clave: tabaquismo, cesación tabáquica, consejo breve, bupropion. Key words: smoking, smoking cessation, brief advine, bupropion. Fuente de financiamiento: Agency for Healthcare Research and Quality, EE.UU.

\section{Comentario}

En Argentina mueren 40.000 personas por año a causa del tabaquismo, de las cuales 6.000 son fumadores pasivos. Se calcula que el tabaco es el producto legal que mata a casi la mitad de sus consumidores habituales.

En este nuevo análisis de la evidencia disponible se distingue que hasta hoy la práctica recomendada en cesación tabáquica es la que se exhorta a realizar desde la atención primaria, ámbito en el cual todo profesional del equipo de salud puede realizar con las herramientas disponibles en nuestro medio una práctica segura y eficaz para lograr el objetivo primario que es que nuestros pacientes dejen de fumar y lo hagan por el mayor tiempo posible ${ }^{1-3}$.

\section{Conclusiones del comentador}

El consejo breve o intensivo utilizados aisladamente o asociado con una buena y segura farmacoterapia como los sustitutos de nicotina, bupropion o la nueva droga vareniclina, serian los tratamientos más respaldados hasta hoy por la evidencia.

Ver glosario*

Guillermo Espinosa [ Medico de Familia. Servicio de Medicina Familiar y Comunitaria y Grupo Anti Tabaco del Hospital Italiano de Buenos Aires. guillermo.espinosa@ hospitalitaliano.org.ar ]

Recibido el 03/07/07 y aceptado el 30/07/07

Espinosa G. Intervenciones en cesación tabáquica. Revisión sistemática de estrategias. Evid. actual. práct. ambul; 10(4):105.Jul-Ago.2007. Comentado de: Ranney L, Melvin C, Lux L, et al. Systematic Review: Smoking Cessation Intervention Strategies for Adults and Adults in Special Populations. Ann Intern Med 2006; 145: 845-856. PMID: 16954352. Disponible en URL: http://www.annals.org/cgi/reprint/145/11/845.pdf último acceso $(30 / 07 / 07)$.

\section{Referencia}

1. Ranney L, et al. Evidence report on tobacco use: prevention, cessation, and control. Evidence report/technology assessment no. 140. Prepared by RTI-UNC evidence-based practice center under contract no. 290-02-0016. Rockville, MD: Agency for Healthcare Research and Quality; 2006. AHRQ Publication 06-E015.

2. Brown R, et al. Cognitive-behavioral treatment for depression in smoking cessation. J Consult Clin Psychol. 2001;69:471-80.

3. Hitsman B, et al. Mahableshwarkar A, Mizes JS, Segraves KA, et al. Antidepressant pharmacotherapy helps some cigarette smokers more than others. J Consult Clin Psychol. $1999 ; 67: 547-54$ 